Homology, Homotopy and Applications, vol.3(2), 2001, pp.385-406

\title{
THE GEOMETRY OF THE LOCAL COHOMOLOGY FILTRATION IN EQUIVARIANT BORDISM
}

\author{
DEV P. SINHA \\ (communicated by Gunnar Carlsson)
}

\begin{abstract}
We present geometric constructions which realize the local cohomology filtration in the setting of equivariant bordism, with the aim of understanding free $G$ actions on manifolds. We begin by reviewing the basic construction of the local cohomology filtration, starting with the Conner-Floyd tom Dieck exact sequence. We define this filtration geometrically using the language of families of subgroups. We then review Atiyah-SegalWilson $K$-theory invariants, which are well-suited for studying the manifolds produced by our techniques. We end by indicating potential applications of these ideas.
\end{abstract}

\section{Introduction}

Local cohomology techniques are an area of rapid development in equivariant topology. Since their introduction by Greenlees $[\mathbf{1 1}]$ they have been used for example to compute the $k u$-homology of classifying spaces of abelian groups [5], they have an interesting relationship with the chromatic filtration [13], and they have been generalized beyond equivariant topology as announced by Dwyer and Greenlees. They give a new duality between homology and cohomology of classifying spaces, which can be played against the Universal Coefficient duality. See [12] for an excellent survey.

One purpose of this note is to better understand the local cohomology filtration by casting it in the language of families. We will see that the local cohomology filtration is coarser than the families filtration. It is more manageable, however, because the algebra which arises is that of localization. Moreover, when one "frees up" the theory, it becomes more computable and gives rise to an interesting relationship between the homology and cohomology, with coefficients in any complex-oriented spectrum (for example ordinary cohomology or $K$-theory), of classifying spaces.

A strong appeal of equivariant bordism as a sub-field of equivariant topology is in its connection with manifold theory. Our geometric constructions allow one to prove collapse results by constructing particular $G$-manifolds which represent classes in the local cohomology spectral sequence. Moreover, we may also bring in Atiyah-Segal-Wilson localized $K$-theory invariants to answer questions about

Received May 14, 2001, revised August 7, 2001; published on September 13, 2001.

2000 Mathematics Subject Classification: Primary: 57R85; Secondary: 55R40, 13D45.

Key words and phrases: cobordism, group homology, local cohomology, plumbing.

(C) 2001, Dev P. Sinha. Permission to copy for private use granted. 
differentials and extensions. On the geometric side, the constructions in this paper are in part an answer to questions about realizing the bordism of classifying spaces geometrically. These questions were posed to the author by Botvinnik who has used such knowledge to solve cases of the Gromov-Lawson-Rosenberg conjecture concerning spin manifolds which admit positive scalar curvature metrics $[\mathbf{3}, \mathbf{2 6}]$. Indeed, the main ideas of this paper came out of conversations with Botvinnik and Sadofsky while the author was visiting the University of Oregon.

This paper will blend well-established ideas of Conner-Floyd, tom Dieck, Atiyah, Segal and Wilson with recent constructions of Greenlees and recent insight of the author to show that the local cohomology filtration is well-suited for addressing questions about both the algebra and geometry associated to free $G$-actions on manifolds. We begin the paper by giving a retrospective of the Conner-Floyd-tom Dieck exact sequence in $\mathbb{Z} / p$-equivariant bordism. We then focus on the case of $(\mathbb{Z} / p)^{2}$, defining the local cohomology filtration. The new insights come when we interpret this filtration in the language of families in order to give a geometric understanding of the filtration. We then show how classes we build using this filtration are well-suited to analysis through the use of Atiyah-Segal-Wilson $K$-theory invariants, taking our time to define these invariants. We close with ways we see in which these techniques could be applied.

Note that Igor Kriz has told us that he has proved collapse of the local cohomology spectral sequence for abelian groups using different methods.

\section{The Conner-Floyd-tom Dieck Exact Sequence}

Conner and Floyd were strongly interested in studying free $G$-actions on manifolds [7]. They saw that bordism theory, newly revolutionized by Thom's work, would give information beyond what homology would see. To that end, they were interested in bordism theory in which representative classes and bordisms were equipped with a free $G$-action. They realized that such a bordism theory was equivalent to the bordism homology of the corresponding classifying space. Throughout we let $E G$ denote a contractible free $G$-space and $B G$ denote the quotient of $E G$ by $G$.

Proposition 2.1. The bordism module of stably complex free $G$-manifolds is isomorphic to $\Omega_{*}^{U}(B G)$.

Proof. Consider the following diagram:

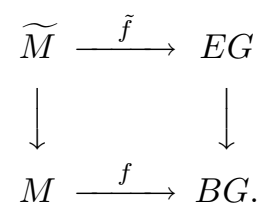

Given a representative $M$ with reference map $f$ to $B G$, pull back the principal $G$ bundle $E G$ to get $\widetilde{M}$, which is in fact a free $G$-manifold. Conversely, starting with a free $G$-manifold $\widetilde{M}$, there is no obstruction to constructing a map $\tilde{f}$ to $E G$. Pass to quotients to obtain $f: M \rightarrow B G$. 
These maps are well-defined, as we apply the previous argument to the manifolds which act as bordisms. The composites of these maps are clearly identity maps.

Suffice it to say that the bordism module $\Omega_{*}^{U}(B G)$ contains a great deal of information about free $G$ actions. It is the main object of study in this paper. It remains mysterious for most groups, being easy to describe only for cyclic groups [20] and having been computed at all for only a few groups, including elementary abelian groups [17]. Conner and Floyd realized that this module is best understood in relation to bordism in which more general actions are allowed. We carefully define this theory now.

We begin by recalling the definition of geometric complex equivariant bordism. Fix $\mathcal{U}_{\mathbb{C}}\left(\mathcal{U}_{\mathbb{R}}\right.$, respectively) a complex (resp. real) representation of which a countably infinite direct sum of any representation of $G$ appears as a summand. Let $B U^{G}(n)$ $\left(B O^{G}(n)\right.$, respectively) be the Grassmanian of complex (resp. real) $n$-dimensional linear subspaces of $\mathcal{U}_{\mathbb{C}}\left(\right.$ resp. $\left.\mathcal{U}_{\mathbb{R}}\right)$, topologized as a direct limit of finite dimensional Grassmanians.

Definition 2.2. A tangentially complex $G$-manifold is a pair $(M, \tau)$ where $M$ is a smooth $G$-manifold and $\tau$ is a lift to $B U^{G}(n)$ of the map to $B O^{G}(2 n)$ which classifies $T M \times \mathbb{R}^{k}$ for some $k$.

We can define bordism equivalence in the usual way to get a geometric version of equivariant bordism.

Definition 2.3. Let $\Omega_{*}^{U, G}$ denote the ring of tangentially complex $G$-manifolds up to bordism equivalence. Let $\Omega^{U, G}(X)$ denote the module (over $\Omega_{*}^{U, G}$ ) of tangentially complex $G$-manifolds equipped with a reference map to $X$ up to bordism equivalence.

Recall that one may prove that geometric bordism theory has suspension isomorphisms or satisfies excision using transversality arguments [21]. Because transversality holds between $G$-manifolds when the range manifold has trivial action, $\Omega_{*}^{U, G}(-)$ has suspension isomorphisms when the $G$-action on the suspension coordinate is trivial. On the other hand, it does not have isomorphisms induced by suspension by (one-point compactified) representations in general, so we say that it is an equivariant homology theory "indexed on the trivial universe" (see [28] for the corresponding statement in terms of spectra).

There is a map $\Omega_{*}^{U}(B G) \rightarrow \Omega_{*}^{U, G}$ defined simply because a free $G$-manifold is a $G$ manifold. Conner and Floyd realized that this map fits in a natural exact sequence. It will be best for us to describe this exact sequence using the language of families, as we will be using this language throughout the paper. There is an excellent discussion of families in [8].

Definition 2.4. A family of subgroups of a group $G$ is a set of subgroups $\mathcal{F}$ such that if $H \in \mathcal{F}$ and $H^{\prime}$ is conjugate to a subgroup of $H$ then $H^{\prime} \in \mathcal{F}$.

Definition 2.5. Let $\mathcal{F}$ be a family of subgroups of $G$. An $\mathcal{F}$-space is a $G$-space all of whose isotropy groups are in $\mathcal{F}$. 
There is a terminal object, unique up to homotopy, in the category of $\mathcal{F}$-spaces which is called $E \mathcal{F}$. In simpler terms, $E \mathcal{F}$ is an $\mathcal{F}$-space which is contractible (after forgetting the $G$-action). For example, if $\mathcal{F}$ is the family consisting of only the identity subgroup, $E \mathcal{F}$ is simply $E G$. We will use the term $\mathcal{F}$-manifold to refer to a manifold which is an $\mathcal{F}$-space.

If $\mathcal{F}_{2} \subset \mathcal{F}_{1}$ are two families, define an $\left(\mathcal{F}_{1}, \mathcal{F}_{2}\right)$-manifold to be $\mathcal{F}_{1}$-manifold with (possible empty) boundary such that its boundary is an $\mathcal{F}_{2}$-manifold.

Definition 2.6. Two $\left(\mathcal{F}_{1}, \mathcal{F}_{2}\right)$-manifolds $M$ and $N$ are bordant when there is an $\left(\mathcal{F}_{1}\right)$-manifold $W$ such that $M \sqcup-N$ is equivariantly diffeomorphic to a codimension zero sub-manifold of $\partial W$ and $\partial W-(M \sqcup N)$ is an $\mathcal{F}_{2}$-manifold.

Let $\Omega_{*}^{U, G}[\mathcal{F}]$ (respectively $\Omega_{*}^{U, G}\left[\mathcal{F}_{1}, \mathcal{F}_{2}\right]$ ) denote the bordism module of tangentially complex $\mathcal{F}$ (resp. $\left(\mathcal{F}_{1}, \mathcal{F}_{2}\right)$ )-manifolds. If $\mathcal{F}_{2} \subset \mathcal{F}_{1}$ are families, there is an inclusion map $\Omega_{*}^{U, G}\left[\mathcal{F}_{2}\right] \rightarrow \Omega_{*}^{U, G}\left[\mathcal{F}_{1}\right]$ as well as a boundary map $\Omega_{*}^{U, G}\left[\mathcal{F}_{1}, \mathcal{F}_{2}\right] \rightarrow$ $\Omega_{*-1}^{U, G}\left[\mathcal{F}_{2}\right]$. There are also inclusion maps for pairs of families, and in particular inclusion maps of the form $\Omega_{*}^{U, G}\left[\mathcal{F}_{1}\right] \rightarrow \Omega_{*}^{U, G}\left[\mathcal{F}_{1}, \mathcal{F}_{2}\right]$, since $\Omega_{*}^{U, G}\left[\mathcal{F}_{1}\right] \cong \Omega_{*}^{U, G}\left[\mathcal{F}_{1}, \phi\right]$, where $\phi$ is the empty family.

Proposition 2.7. Let $\mathcal{F}_{3} \subset \mathcal{F}_{2} \subset \mathcal{F}_{1}$ be three families of subgroups of $G$. The sequence

$$
\cdots \rightarrow \Omega_{*}^{U, G}\left[\mathcal{F}_{2}, \mathcal{F}_{3}\right] \rightarrow \Omega_{*}^{U, G}\left[\mathcal{F}_{1}, \mathcal{F}_{3}\right] \rightarrow \Omega^{U, G}\left[\mathcal{F}_{1}, \mathcal{F}_{2}\right] \stackrel{\partial}{\rightarrow} \Omega_{*-1}^{U, G}\left[\mathcal{F}_{2}, \mathcal{F}_{3}\right] \rightarrow \cdots
$$

is exact.

This exact sequence is essentially the long exact sequence of a triple. In fact, one may deduce it from the long exact sequence of a triple by showing that $\Omega_{*}^{U, G}[\mathcal{F}] \cong$ $\Omega_{*}^{U, G}(E \mathcal{F})$ and $\Omega_{*}^{U, G}\left[\mathcal{F}_{1}, \mathcal{F}_{2}\right] \cong \Omega_{*}^{U, G}\left(E \mathcal{F}_{1}, E \mathcal{F}_{2}\right)$.

We now specialize to the case $G=\mathbb{Z} / p$, for which there are only three families: the empty family, the family of the trivial (identity) subgroup which we call $e$, and the family of all subgroups which we call $\mathcal{A}$. We apply Proposition 2.7 using these three families and identify the terms. Note that $\Omega_{*}^{U, G}[\mathcal{A}, \phi]=\Omega_{*}^{U, G}$. Next consider $\Omega_{*}^{U, G}[e, \phi]$. By definition this is the bordism module of free $G$-manifolds, which we have identified in Proposition 2.1, and the map from it to $\Omega_{*}^{U, G}$ is simply the forgetful map. Hence the exact sequence reads as follows.

$$
\cdots \rightarrow \Omega_{*}^{U}(B G) \stackrel{i}{\rightarrow} \Omega_{*}^{U, G} \stackrel{j}{\rightarrow} \Omega_{*}^{U, G}[\mathcal{A}, e] \stackrel{\partial}{\rightarrow} \Omega_{*-1}^{U}(B G) \rightarrow \cdots
$$

Conner and Floyd went further in $[\mathbf{7}]$ to identify $\Omega_{*}^{U, G}[\mathcal{A}, e]$. The map $j$ can be interpreted in terms of "reduction to fixed sets". The relevant general fact is as follows.

Proposition 2.8. An $\left(\mathcal{F}_{1}, \mathcal{F}_{2}\right)$-manifold $M$ is bordant to any smooth neighborhood $\mathcal{N}\left(\bigcup_{H \notin \mathcal{F}_{2}} M^{H}\right)$ of the locus of points in $M$ fixed by subgroups not in $\mathcal{F}_{2}$.

Proof. Let $W=M \times[0,1]$, with "straightened corners". Then $\partial W$ is an $\mathcal{F}_{2}$ manifold outside of the codimension zero submanifolds $M \times 0$ and a tubular neighborhood of $\left(\bigcup_{H \notin \mathcal{F}_{2}} M^{H}\right) \times 1$, so $W$ is the required bordism. 
When $G=\mathbb{Z} / p$, tubular neighborhoods of fixed sets are diffeomorphic to $G$ vector bundles over those fixed sets, so Conner and Floyd were able to identify $\Omega_{*}^{U, G}[\mathcal{A}, e]$ in terms of bordism modules of manifolds with trivial $G$-action which equipped with $G$-vector bundles.

Returing to our original question, namely as to the structure of $\Omega_{*}^{U}(B G)$, we see that this exact sequence gives rise to two-stage filtration into the free $G$-manifolds which are the boundaries of general $G$-manifolds (the cokernel of $\partial$ ) and those which are not (the image of $i$ ). We may now state that a main goal of this paper is to give a similar description of the filtration on $\Omega_{*}^{U}(B G)$ for more general $G$ which arises from Greenlees's local cohomology machinery. First, however, we wish to gain computational understanding from our current exact sequence.

Unfortunately, to this day the geometric theories $\Omega_{*}^{U, G}$ are mysterious, with partial knowledge for abelian $G$ and a complete computation for $\mathbb{Z} / 2$ which may be deduced from [25]. Hence it is not computationally useful to study $\Omega_{*}^{U}(B G)$ using this exact sequence. To make this exact sequence more algebraically manageable requires two steps.

The first step was taken by tom Dieck in [9], who realized that Conner and Floyd's exact sequence was related to localization methods in equivariant $K$-theory being developed at the time by Atiyah and Segal [2]. To make this connection, tom Dieck defined a more homotopy theoretic version of equivariant bordism. He crafted a spectrum $M U^{G}$, analogous to $M U$, whose corresponding infinite loop space is $\lim _{V} \operatorname{Maps}\left(S^{n \oplus V}, T\left(\xi_{|V|}^{G}\right)\right)^{G}$, where $V$ ranges over isomorphism classes of complex representations of $G, S^{n \oplus V}$ is the one-point compactification of the Whitney sum of $\mathbb{C}^{n}$ with trivial $G$ action and $V$, and $T\left(\xi_{|V|}^{G}\right)$ is the Thom space of the universal complex $G$-bundle. See [8] for a recent treatment of tom Dieck's construction. Though this spectrum has been defined for thirty years and studied actively, it is only recently that its coefficients have been understood in any cases $[\mathbf{1 8}, \mathbf{2 5}]$.

The equivariant homology and cohomology theories corresponding to this spectrum have suspension isomorphisms with respect to any representation. Moreover, the cohomology theory has a Thom isomorphism for complex $G$-vector bundles, and so has some theory of characteristic classes. An interesting class of $G$-vector bundles are complex representations $V$, considered as bundles over a point. The associated Euler classes are denoted $e_{V} \in M U_{G}^{m}(p t)=.M U_{-m}^{G}$. There is a Pontryagin-Thom map from $\Omega_{*}^{U, G}$ to $M U_{*}^{G}$, but it is not an isomorphism, since transversality arguments fail in an equivariant setting [9] (in particular the Euler classes $e_{V}$ are non-zero classes in negative homological degrees where geometric bordism is zero by definition - see $[\mathbf{8}]$ ).

Reflecting on the Conner and Floyd exact sequence for $G=\mathbb{Z} / p$, tom Dieck considered the cofiber sequence

$$
E G_{+} \rightarrow S^{0} \rightarrow \widetilde{E G},
$$

whose long exact sequence in $\Omega^{U, G}$ theory is the Conner-Floyd exact sequence. We say that the resulting long exact sequence in $M U^{G}$ theory is the tom Dieck exact sequence.

tom Dieck realized that the map from $M U_{*}^{G}$ to $M U_{*}^{G}(\widetilde{E G})$ had an interpretation 
in terms of localization (and hence a connection with the work of Atiyah and Segal $[2])$. The key observation is that one model for $E G$ for cyclic $G$ is as the unit sphere in the representation $\oplus_{\infty} V$ which we denote $S\left(\oplus_{\infty} V\right)$, where $V$ is the standard representation of $G$ as the roots of unity in the complex numbers. Hence a model for $\widetilde{E G}$ is the one-point compactification of that representation, which we denote $S^{\infty V}$.

For any commutative ring $R$ and element $e \in R$ let $R\left[e^{-1}\right]$ denote the localization of $R$ obtained by inverting $e$.

Lemma 2.9. For any $G, \widetilde{M U_{*}^{G}}\left(S^{\oplus \infty}\right) \cong M U_{*}^{G}\left[e_{V}^{-1}\right]$ as rings.

Proof. The left-hand side $\widetilde{M U_{*}^{G}}\left(S^{\oplus_{\infty} V}\right)$ is a ring because $S^{\oplus_{\infty} V}$ is an $H$-space through the equivalence

$$
S^{\oplus \infty} V \wedge S^{\oplus_{\infty} V} \cong S^{\oplus \infty} V .
$$

To compute the left-hand side, apply $\widetilde{M U_{*}^{G}}$ to the identification $S^{\oplus_{\infty} V}=\underline{\lim } S^{\oplus_{n} V}$. After applying the suspension isomorphisms $\widetilde{M U_{*}^{G}}\left(S^{\oplus_{k} V}\right) \cong \widetilde{M U_{*+|V|}^{G}}\left(S^{\oplus_{k+1} V}\right)$, the maps in the resulting directed system are multiplication by the $e_{V}$.

tom Dieck also uses the fact that transversality arguments go through in the presence of free $G$ actions to show that $M U_{*}^{G}(E G) \cong M U_{*}(B G)$. Here one could also use Adams's transfer argument [1] to show $\left(M U^{G} \wedge E G_{+}\right)^{G} \cong M U \wedge B G_{+}$. Hence the tom Dieck exact sequence reads

$$
\cdots \rightarrow M U_{*}(B G) \rightarrow M U_{*}^{G} \rightarrow M U_{*}^{G}\left[e_{\rho}^{-1}\right] \rightarrow \cdots .
$$

Moreover, just as Conner and Floyd computed the third term in the geometric setting, one can compute the localization $M U_{*}^{G}\left[e_{\rho}{ }^{-1}\right]$. The fact that one can compute such a localization is the starting point for the computation of $M U_{*}^{G}$ for abelian $G$ [25], which reveal that the structure of these rings is quite complicated. In fact, their structure is complicated to an extent which renders the tom Dieck exact sequence not usable for computation of $M U_{*}(B G)$.

The next step one must take to make this exact sequence more computable is to "free up" the spectrum $M U^{G}$. Consider the mapping spectrum $\operatorname{Maps}\left(E G_{+}, M U^{G}\right)$. Its homotopy groups are the homotopy groups of the $G$-maps from $E G_{+}$to $M U^{G}$. Because $E G_{+}$is free, we have

$$
\operatorname{Maps}^{G}\left(E G_{+}, M U^{G}\right)=\operatorname{Maps}\left(B G_{+}, i_{*}\left(M U^{G}\right)\right)=\operatorname{Maps}\left(B G_{+}, M U\right)
$$

where $i_{*}$ takes a $G$-spectrum and passes to the underlying spectrum. Note as well that because $E G_{+}$is simply $S^{0}$ non-equivariantly we have that $i_{*}\left(\operatorname{Maps}\left(E G_{+}, M U^{G}\right)\right)=M U$ as well. Hence by Adams's transfer argument [1] we have that

$$
E G_{+} \wedge_{G} \operatorname{Maps}\left(E G_{+}, M U^{G}\right)=B G_{+} \wedge i_{*}\left(\operatorname{Maps}\left(E G_{+}, M U^{G}\right)\right)=M U \wedge B G_{+} .
$$

Finally, let $t^{G} M U^{G}$ denote the spectrum $\operatorname{Maps}\left(E G_{+}, M U^{G}\right) \wedge \widetilde{E G}$. For cyclic $G$ the coefficients of $t^{G}$ are obtained by inverting the Euler class of the standard representation, as in Lemma 2.9, in the coefficients of $\operatorname{Maps}\left(E G_{+}, M U^{G}\right)$ which 
again are just $M U^{*}(B G)$. Thus if we smash the spectrum $\operatorname{Maps}\left(E G_{+}, M U^{G}\right)$ with tom Dieck's cofiber sequence and take homotopy groups we get

$$
\cdots \rightarrow M U_{*}(B G) \rightarrow M U^{*}(B G) \rightarrow M U^{*}(B G)\left[e_{\rho}{ }^{-1}\right] \rightarrow \cdots
$$

The terms in this exact sequence are all computable. Landweber used the Gysin sequence for the fiber bundle $S^{1} \rightarrow B \mathbb{Z} / p \rightarrow \mathbb{C} P^{\infty}$ to show in [19] that $M U^{*}(B \mathbb{Z} / p) \cong M U^{*}[[x]] /[p]_{F}(x)$ where $[p]_{F}(x)$ is the $p$-series in the formal group law over $M U$ (see [23]). More topologically, $p_{F}(x)$ is the Euler class of the $p$ th tensor power of the tautological bundle over $\mathbb{C} P^{\infty}$, whose associated sphere bundle is the fiber bundle above. Hence we may finally be explicit about the exact sequence, which reads

$$
\cdots \rightarrow M U_{*}(B G) \rightarrow M U^{*}[[x]] /[p]_{F}(x) \rightarrow M U^{*}((x)) /[p]_{F}(x) \rightarrow \cdots,
$$

where in general we use $R((x))$ to denote $R[[x]]\left[x^{-1}\right]$. We may now compute $M U_{*}(B G)$, as was first done by Landweber [20]. We let the unit class in $M U_{*}(B G)$ denote the class in $M U_{*}(B G)$ which is the image of 1 under the map from $M U_{*}$ induced by inclusion of a point into $B G$.

Theorem 2.10. $M U_{*}(B \mathbb{Z} / p)$ is generated as a module by the unit class and by classes $y_{i}$ with relations $p y_{i}+a_{1} y_{i-1}+\cdots+a_{i-1} y_{1}=0$, where $a_{i}$ is the coefficient of $x^{i}$ in the series $p_{F}(x)$.

Proof. We compute the cokernel and kernel of the map $M U^{*}[[x]] /[p]_{F}(x) \rightarrow$ $M U^{*}((x)) /[p]_{F}(x)$ in the sequence of Diagram 1 above. The cokernel is generated by the negative powers of $x$. Let $y_{i}$ denote the image of $x^{-i}$ in $M U_{*}(B G)$. By multiplying the $p$-series by negative powers of $x$, we have that

$$
p x^{-i}+a_{1} x^{1-i}+\cdots+a_{i-1} x+\text { non-negative powers of } \mathrm{x}=0,
$$

so we deduce the stated relation. Clearly all relations arise from multiplying the $p$-series by a Laurent polynomial in $x$, so these relations suffice to generate all relations.

The kernel is generated by the series $p_{F}(x) / x$. It is a free $M U_{*}$ module on one generator.

Because the cokernel consists entirely of $M U_{*}$-torsion ( $p$-torsion, in fact) and the kernel is free, we see that $M U_{*}(B G)$ splits as this sum of these modules. Moreover, since the ideal generated by the unit class is non-zero and free, it must map isomorphically to its image under $i$, namely the ideal generated by $p_{F}(x) / x$.

It is interesting to note that the Atiyah-Hirzebruch spectral sequence to compute this module has as many non-trivial extensions as possible.

To summarize the exact sequences we have introduced, for $G=\mathbb{Z} / p$ we have a commutative diagram as follows. 


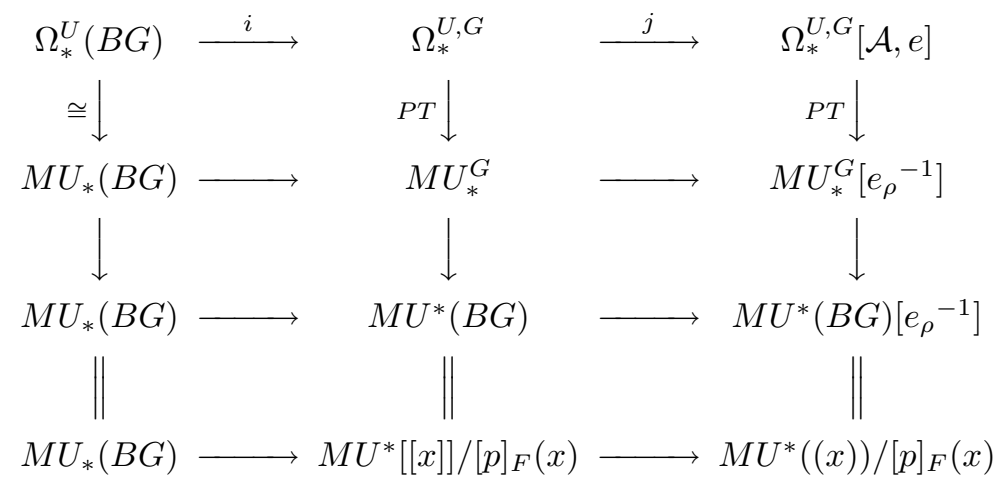

The middle two rows of this diagram are known as the Tate diagram [15].

We may use this commutative diagram to give geometric representatives of the classes in $\Omega_{*}^{U}(B G)$. The key observation is the following.

Proposition 2.11 (Lemma 3.1 of [8]). The class $e_{\rho}^{-1} \in M U_{*}^{G}\left[e_{\rho}{ }^{-1}\right]$ is the image under the Pontryagin-Thom map of $D(\rho)$, the unit disk in the representation $\rho$, which is a $G$-manifold with $\mathcal{A}$-boundary.

Sketch of Proof. The appropriate setting for the proof is that of "stable manifolds". Given a manifold with boundary $M, \partial M$ and a map of pairs from this manifold to $D(V), S(V)$ for some representation $V$ there is a Pontryagin-Thom map which produces an element of $\pi_{\operatorname{dim} M-\operatorname{dim} V}\left(M U^{G}\right)$. Non-equivariantly, such a map does not produce any classes beyond those produced by the standard Pontryagin-Thom map (it cannot, since the usual Pontryagin-Thom map is an isomorphism). Equivariantly, this procedure does produce new classes, in fact giving all of $M U_{*}^{G}$ [4].

For example, $e_{V}$ is the image under this map of the stable manifold in which $M$ is a point which maps to $0 \in D(V)$. It is then conceivable that the inverse of this class in the localization is given by the image under the Pontryagin-Thom map of $D(V)$ mapping to a point (zero-disk).

Corollary 2.12. The generator $y_{i} \in \Omega_{2 i-1}^{U}$ is represented by $S\left(\oplus_{i} \rho\right)$.

It is quite straightforward to geometrically understand the unit class in $M U_{*}(B G)$ as well by running through the isomorphism of Proposition 2.1.

Proposition 2.13. The unit class in $M U_{*}(B G)$ is represented by $G$ itself, as a zero-dimensional manifold.

Hence, we not only have an algebraic computation of $\Omega_{*}^{U}(B G)$ but we have explicit geometric understanding of most of the generators as (quotients of) free $G$ manifolds which are boundaries of arbitrary $G$-manifolds. Note that having a free $G$-manifold as the boundary of an arbitrary $G$-manifold is precisely the setting for studying the free $G$-manifold using the $K$-theoretic invariants of Atiyah and Segal $[\mathbf{2}]$ as further developed by Wilson [29]. We will develop these ideas below. 
Our goal for more general $G$ is to develop similar understanding of $\Omega_{*}^{U}(B G)$, with as much control as possible over both the algebra and the geometry. We will outline a program for doing so in this paper, describing a computational tool which generalizes the discussion of this section, namely the local cohomology filtration. There are in fact two ways in which one may generalize the exact sequences developed in this section, the other known as the families filtration, which is described very well in [8]. Briefly, one filters $E G$ by a sequence of maps $E G \rightarrow E \mathcal{F}_{1} \rightarrow \cdots E \mathcal{F}_{n}$, where $F_{i+1}$ is obtained from $F_{i}$ by adding one (conjugacy class of) subgroup. For many theories such as bordism, the equivariant homology of the cofiber of $E \mathcal{F}_{i} \rightarrow E \mathcal{F}_{i+1}$ is equivalent to (non-equivariant) homology of some sort of classifying space (much as in Proposition 2.1). The local cohomology filtration is coarser than this filtration but surprisingly much more computable once one "frees up" the theories involved.

\section{The Local Cohomology Filtration}

Just as the survey $[\mathbf{6}]$ successfully illustrated equivariant theory by concentrating on $\mathbb{Z} / 2$, we are going to focus on $(\mathbb{Z} / p)^{2}$, giving an indication of how things would generalize as we go along.

In the last section it was useful for compuations to notice that the third term Conner-Floyd-tom Dieck sequence, namely the one associated to $S^{\oplus_{\infty} \rho}$, is a localization. To continue to have localizations involved in our computations, we are going to assemble $E G$ from pieces of the form $S^{\oplus \infty} V$ for various $V$.

For $G=(\mathbb{Z} / p)^{2}, E G$ is no longer given by an action on a sphere but on a product of spheres. Let $G$ be generated by elements $x$ and $y$ (so that $p x=p y=x+y-x-y=$ 0 ). Let $V_{x}$ (respectively $V_{y}$ ) be the standard representation of $\mathbb{Z} / p$ pulled back to $G$ through its projection to $G /\langle x\rangle$ (respectively $G /\langle y\rangle$ ). We have that

$$
E G=S\left(\oplus_{\infty} V_{x}\right) \times S\left(\oplus_{\infty} V_{y}\right) .
$$

Hence we smash the cofiber sequence $S\left(\oplus_{\infty} V_{x}\right)_{+} \rightarrow S^{0} \rightarrow S_{\infty}^{\oplus_{x}}$ with the correspondence sequence with $V_{x}$ replaced by $V_{y}$ to get a commutative diagram as follows:

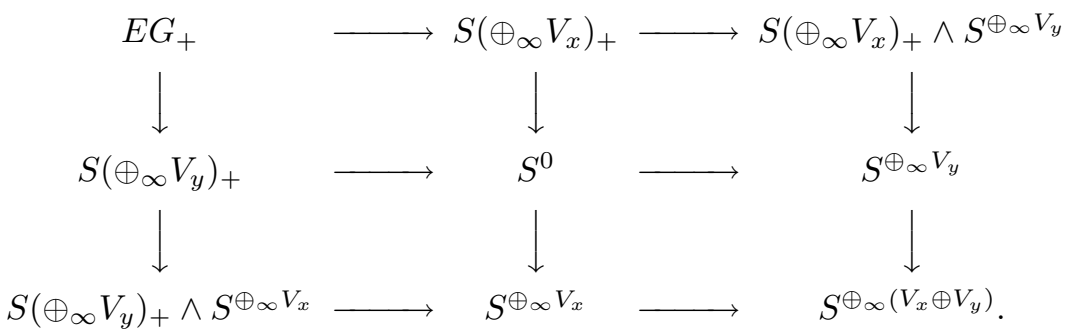

In this situation, $\Sigma^{2} E G_{+}$is known as the total cofiber of the square diagram consisting of the last two spaces of the last two rows in the above diagram. All of the spaces in question, except for $S^{0}$, are of the form $S^{\oplus_{\infty} V}$ as desired.

We pause for a moment to recall basic definitions of cubical diagrams and their total cofibers (from [10]) and give a canonical filtration on those total cofibers. 
Let $\underline{n}=\{1, \cdots, n\}$ and let $\mathcal{P}(\underline{n})$ denote the category of subsets of $\underline{n}$ with morphisms given by inclusion. A cubical diagram of spaces (of dimension $n$ ) is a functor from $\mathcal{P}(\underline{n})$ to based spaces. We often let $X$ • denote such a functor, $X_{S}$ denote the value of this functor on $S \in \operatorname{ob}(\mathcal{P}(\underline{n}))$ and $f_{S, S^{\prime}}$ denote the unique morphism from $X_{S}$ to $X_{S^{\prime}}$ when $S \subseteq S^{\prime}$. A morphism of cubical diagrams is defined as usual for a diagram (or functor) category.

Cubical diagrams are a convenient diagram category in part because a morphism of cubical diagrams may be viewed as a cubical diagram of dimension greater by one. Let $i$ be an element of $\underline{n+1}$ and by let $\varphi_{i}$ denote the order-preserving inclusion from $\underline{n}$ to $n+1$ for which $i$ is not in the image. We may define an $n+1$-dimensional cubical diagram $Z_{\bullet}$ from a morphism $X_{\bullet} \rightarrow Y_{\bullet}$ by letting $Z_{\varphi_{i}(S)}=X_{S}, Z_{\varphi_{i}(S) \cup i}=Y_{S}$ and the map from $Z_{\varphi_{i}(S)}$ to $Z_{\varphi_{i}(S) \cup i}$ be the map from $X_{S}$ to $Y_{S}$. Conversely, given a cubical diagram of dimension $n$ one may view it as a morphism of cubical diagrams of dimenstion $n-1$ in $n$ ways.

We now define the total cofiber of a cubical diagram, which is a functor from cubical diagrams to spaces we will use extensively. For a cube $I^{n}$ whose vertices are naturally labelled by subsets of $\underline{n}$ let $\partial_{S} I^{n}$ for a subset $S$ of $\underline{n}$ denote the face whose vertices are subsets $S$.

Definition 3.1. The total cofiber of an $n$-dimensional cubical diagram $X$. is the quotient of the union $\amalg X_{S} \times \partial_{\underline{n}-S} I^{n}$ through the identifications

- For $x \in X_{S}$ and $S \subset S^{\prime}, x \times \partial_{\underline{n}-S^{\prime}} I^{n} \subset x \times \partial_{\underline{n}-S} I^{n}$ is identified with $f_{S, S^{\prime}}(x) \times$ $\partial_{\underline{n}-S^{\prime}} I^{n}$.

- Points of the form $* \times \partial_{A} I^{n}$, where $*$ is the basepoint of $X_{S}$ and $A$ is a face which does not meet the initial vertex (labelled by the empty set), are all identified to a basepoint.

There are Puppe sequences for cubical diagrams. Given a map of cubes $X_{\bullet} \rightarrow$ $Y_{\bullet}$ one may take the Puppe sequence for each map $X_{S} \rightarrow Y_{S}$. These sequences fit together to define a sequence of cubical diagrams which we also call a Puppe sequence. Moreover, we have the following.

Proposition 3.2. Let $C_{k}$ be the total cofiber of the $k$ th cube in the Puppe sequence of a map $X_{\bullet} \rightarrow Y_{\bullet}$. The sequence $C_{0} \rightarrow C_{1} \rightarrow \cdots$ is a sequence of spaces weakly equivalent to the Puppe sequence associated to the induced map from the total cofiber of $X \bullet$ to the total cofiber of $Y_{\bullet}$.

There is a canonical filtration on the total cofiber of a cubical diagram.

Proposition 3.3. Let $h$ be a homology theory and let $X$. be a cubical diagram of dimension $n$. There is an $n$-column spectral sequence whose $E_{1}$ term is given by

$$
E_{p, q}^{1}=\bigoplus_{\substack{S \subset \underline{n} \\ \# S=n-p}} h_{q-\# S}\left(X_{S}\right)
$$

and

$$
\left.d_{1}\right|_{h_{*}\left(X_{S}\right)}=\Sigma_{S^{\prime} \supset S}(-1)^{\# S^{\prime} \cdot \mu\left(S, S^{\prime}\right)}\left(f_{S, S^{\prime}}\right)_{*},
$$


where $\mu\left(S, S^{\prime}\right)$ is the element of $S^{\prime}$ not in $S$, which converges to the homology of the total cofiber of $X_{\bullet}$.

Partial proof. We supply a proof, which is immediately generalizable, in the case of square diagrams.

Consider the sequence of square diagrams

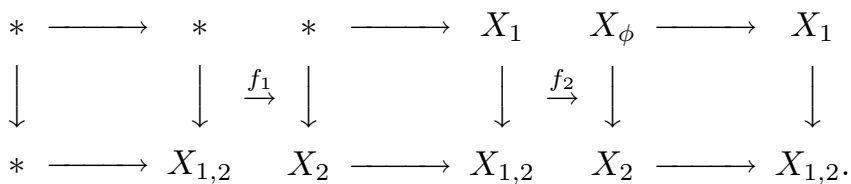

These maps give rise to a sequence of maps between total cofibers of those diagrams, which we denote $T_{0} \stackrel{f_{1}}{\rightarrow} T_{1} \stackrel{f_{2}}{\rightarrow} T_{2}$. As there is for any such sequence of maps, there is a three-column spectral sequence whose $E_{1}$ term consists of the homology of $T_{0}$, the cofiber of $f_{1}$ which we denote $\operatorname{cof}\left(f_{1}\right)$, and $\operatorname{cof}\left(f_{2}\right)$. Let $g_{i}$ denote the map from $T_{i}$ to $\operatorname{cof}\left(f_{i}\right)$ (with $g_{0}$ the identity map by convention) and $\partial_{i}$ the map in the Puppe sequence from $\operatorname{cof}\left(f_{i}\right)$ to $\Sigma T_{i-1}$. The $d_{1}$ of this spectral sequence is given by $g_{i-1} \circ \partial_{i}$. This spectral sequence converges to the homology of $T_{2}$.

We have that $T_{0}$ is simply $X_{1,2}$. Next we see that the cofiber of the map from $T_{0}$ to $T_{1}$ is the total cofiber of the cube defined by $f_{1}$ as a map of squares. This total cofiber is in turn the total cofiber of the square

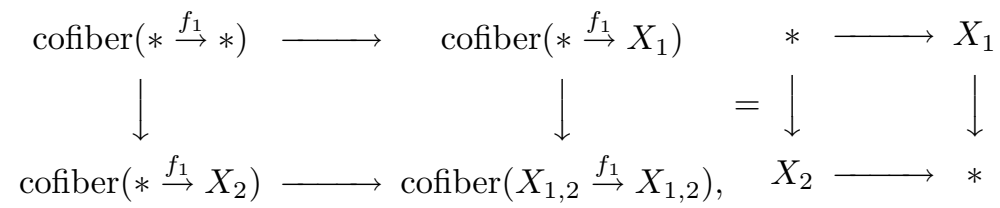

which is simply $\Sigma X_{1} \wedge \Sigma X_{2}$. Similarly, the cofiber of the map from $T_{1}$ to $T_{2}$ is $\Sigma^{2} X_{\phi}$.

Finally, we identify the $d_{1}$ differential. As we stated above, it suffices to understand the boundary maps in the Puppe sequence of total cofibers. By Proposition 3.2, we may instead look at the Puppe sequence of cubes. Unraveling the definitions, we see that the boundary maps used to define $d_{1}$ in a spectral sequence of a filtration are a wedge of suspensions of structure maps from the original cube, with signs introduced by the interchange of suspension coordinates.

We apply this proposition to the square diagram

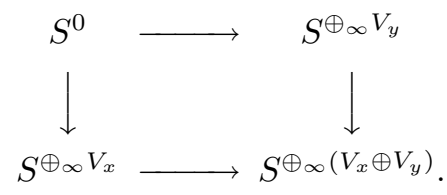

where the homology theory in question is either $M U^{G}$ or respectively $\operatorname{Maps}\left(E G_{+}, M U^{G}\right)$, and make identifications using Lemma 2.9 in order to deduce the following theorem originally due to Greenlees. Once again we restrict our attention to the case of $(\mathbb{Z} / p)^{2}$. 
Theorem 3.4 (Greenlees). There are three-column spectral sequences converging to $M U_{*}\left(B\left(\mathbb{Z} / p^{2}\right)\right)$ whose $E^{1}$ terms are given by

$$
M U_{*}^{G}\left[e_{V_{x}}^{-1}, e_{V_{y}}^{-1}\right] \stackrel{+,-}{\longleftarrow} M U_{*}^{G}\left[e_{V_{x}}^{-1}\right] \oplus M U_{*}^{G}\left[e_{V_{y}}^{-1}\right] \longleftarrow M U_{*}^{G},
$$

and

$$
\begin{aligned}
& M U_{*}[[x, y]]\left[(x y)^{-1}\right] /\left(p_{F}(x), p_{F}(y)\right) \\
& \stackrel{+,-}{\longleftarrow} M U_{*}[[x, y]]\left[x^{-1}\right] /\left(p_{F}(x), p_{F}(y)\right) \oplus M U_{*}[[x, y]]\left[y^{-1}\right] /\left(p_{F}(x), p_{F}(y)\right) \\
& \left.\longleftarrow M U^{*}(B G)=M U_{*}[[x, y]] /\left(p_{F}(x)\right), p_{F}(y)\right),
\end{aligned}
$$

where the grading defined so that these columns are in degrees $-2,-1$ and 0 and where the maps above are the canonical maps arising from the fact that each of the columns, which are graded rings, is obtained from the next by localization.

This spectral sequence is called the local cohomology spectral sequence, because in fact the $E^{2}$ term is precisely what is known as the local cohomology of the ring $M U_{*}^{G}$, respectively $M U^{*}(B G)$, at the ideal generated by the Euler classes $e_{V_{x}}$ and $e_{V_{y}}[\mathbf{1 6}]$. The algebra of local cohomology and its relevance to this situation is welldocumented in the work of Greenlees and his collaborators $[\mathbf{1 1}, \mathbf{1 2}, \mathbf{1 4}]$. In short, local cohomology groups of a ring $R$ at an ideal $I$ are the derived functors of the $I$ torsion functor. Because of its connection with well-developed homological algebra, the local cohomology filtration has lead to many advances in equivariant topology, and the topology of classifying spaces in particular.

\section{A Geometric Version of the Local Cohomology Filtration}

Here we will take a geometric approach by recasting this spectral sequence in the language of families. First we replace our diagram of nine spaces from Diagram 2 - the cofiber sequence of cofiber sequences - with a diagram of bordism modules defined by families. Then, we give geometric definitions of the differentials in this spectral sequence. Such analysis leads to conditions for collapse of the spectral sequence, which we verify for $G=(\mathbb{Z} / p)^{2}$.

Let $e$ and $\mathcal{A}$ continue to denote the families (of subgroups of $(\mathbb{Z} / p)^{2}$ now) consisting of only the trivial group and of all subgroups, respectively. By abuse, let $\langle x\rangle$ denote the family consisting of the subgroup generated by $x$ along with the trivial subgroup, and similarly for $\langle y\rangle$. Let $\langle x\rangle \cup\langle y\rangle$ denote the family consisting of the subgroups generated by $x$ and by $y$ along with the trivial subgroup.

Consider the following diagram of bordism modules. 


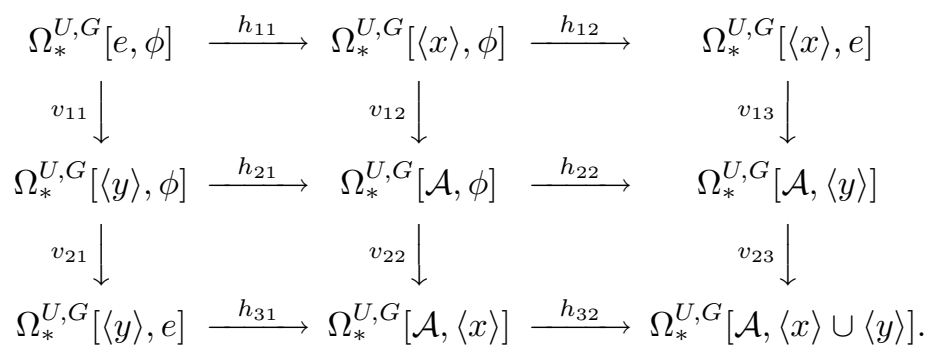

There are boundary maps which continue the sequences defined by the first two rows and columns. For example, $h_{13}: \Omega_{*}^{U, G}[\langle x\rangle, e] \rightarrow \Omega_{*-1}^{U, G}[e, \phi]$ by defined by taking the boundary of a representative class. These are simply the boundary maps of Proposition 2.7. There are also boundary maps for the last column and row. Given an $(\mathcal{A},\langle x\rangle \cup\langle y\rangle)$-manifold $M$ representing a class in bordism, the boundary map of the last column sends $[M]$ to $\left[\nu\left((\partial M)^{x}\right)\right]$ the class represented by a tubular neighborhood (in $\partial M$ ) of the $x$-fixed set of $\partial M$.

Proposition 4.1. The rows and columns of Diagram 4, continued with the boundary maps defined above, form long exact sequences.

For the first and second rows and columns this is an application Proposition 2.7. For the last row and column, the verification is similar to that of the long exact sequence of a pair in bordism.

As mentioned above, we are constructing an analog of Diagram 2.

Proposition 4.2. The sequences of Diagram 4 map to those of Diagram 2 through the Pontryagin-Thom map.

Our cubical diagram formalism allows us to use Diagram 4 to construct an analog of the local cohomology spectral sequence.

Theorem 4.3. There is a spectral sequence whose $E^{1}$ term is given by

$$
\Omega_{*}^{U, G}[\mathcal{A},\langle x\rangle \cup\langle y\rangle] \stackrel{v_{23}-h_{32}}{\longleftarrow} \Omega_{*}^{U, G}[\mathcal{A},\langle y\rangle] \oplus \Omega_{*}^{U, G}[\mathcal{A},\langle x\rangle] \stackrel{h_{22} \oplus v_{22}}{\longleftarrow} \Omega_{*}^{U, G}[\mathcal{A}, \phi]=\Omega_{*}^{U, G},
$$

which converges to $M U_{*}(B G)$. This spectral sequence maps to those of Theorem 3.4.

Because the maps in this spectral sequence are geometrically defined we may further our understanding of how the various sub-quotients in the local cohomology filtration give rise to classes in $M U_{*}(B G)$.

For example, we see that the module $\Omega_{*}^{U, G}[\mathcal{A},\langle x\rangle \cup\langle y\rangle]$ maps to $\Omega_{*}^{U, G}[e, \phi]=$ $M U_{*}(B G)$ by sending a class $[M]$ to $\left[\partial\left(\nu(\partial M)^{x}\right)\right]$, which is the boundary of a tubular neighborhood (in $\partial M$ ) the the $x$-fixed set of $\partial M$. We may verify this by starting at $(\mathcal{A},\langle x\rangle \cup\langle y\rangle)$ and composing a verticle arrow with a horizontal arrow in Diagram 4. If $M_{1}=\partial W_{1}$ and $M_{2}=\partial W_{2}$ are free $\mathbb{Z} / p$-manifolds which bound arbitrary $\mathbb{Z} / 2$-manifolds, then $W_{1} \times W_{2}$ is naturally a $(\mathbb{Z} / p)^{2}$-manifold with boundary which represents a class in $\Omega_{*}^{U, G}[\mathcal{A},\langle x\rangle \cup\langle y\rangle]$. Under the map to $M U_{*}(B G)$, this class maps to $\left[M_{1} \times M_{2}\right]$. 
We may understand the $d_{2}$ differential as the map defined by $h_{12}$ applied to a lift of a class $[M] \in \Omega_{*}^{U, G}[\mathcal{A}, \phi]$ which maps to zero under $v_{22}$ and $h_{22}$. Geometrically, this differential determines whether a manifold $[M]$ which is cobordant to both a manifold with no $\langle x\rangle$-fixed points and to another with no $\langle y\rangle$-fixed points is actually cobordant to a free manifold.

Of course, in order to prove that a differential is zero it suffices to find non-zero classes in $M U_{*}(B G)$ to which the classes in the spectral sequence coincide.

Theorem 4.4. The spectral sequence of Diagram 3 in Theorem 3.4 collapses at $E^{2}$.

Proof. The kernel of the $d_{1}$ map

$$
\begin{gathered}
M U_{*}[[x, y]] /\left(p_{F}(x)\right) \rightarrow M U_{*}[[x, y]]\left[x^{-1}\right] /\left(p_{F}(x), p_{F}(y)\right) \oplus \\
M U_{*}[[x, y]]\left[y^{-1}\right] /\left(p_{F}(x), p_{F}(y)\right)
\end{gathered}
$$

is the ideal generated by the series $p_{F}(x) / x \cdot p_{F}(y) / y$. We claim that this series is in the image of the map from $M U_{*}(B G)$ to this quotient in the filtration, and hence cannot support a $d_{2}$ differential. For dimensional reasons, no other differentials are possible.

The class which maps to the series $p_{F}(x) / x \cdot p_{F}(y) / y$ is the unit class in $M U_{*}(B G)$, represented by $G$ itself, a zero-dimensional manifold. This follows from the fact that the unit class in $M U_{*}(B \mathbb{Z} / p)$ mapped to $p_{F}(x) / x$ in $M U^{*}(B \mathbb{Z} / p)$ in the Tate sequence, as we established in Theorem 2.10.

Perhaps the most fun and interesting aspect of this analysis occurs for in the middle column of the spectral sequence, even though it cannot support a differential simply for dimensional reasons. If two classes $[M] \in \Omega_{*}^{U, G}[\mathcal{A},\langle y\rangle]$ and $[N] \in \Omega_{*}^{U, G}[\mathcal{A},\langle x\rangle]$ have the same image in $\Omega_{*}^{U, G}[\mathcal{A},\langle x\rangle \cup\langle y\rangle]$ then if we take $[\partial M] \in \Omega_{*}^{U, G}[\langle y\rangle, \phi]$ it must go to zero under $v_{21}$ and hence lift to $\Omega_{*}^{U, G}[e, \phi]=M U_{*}(B G)$.

Geometrically, becuase $[M]$ and $[N]$ have the same image in $\Omega_{*}^{U, G}[\mathcal{A},\langle x\rangle \cup\langle y\rangle]$ it means that there is a cobordism $W$ between a tubular neighborhood of $\bigcup_{H \neq\langle x\rangle \text { or }\langle y\rangle} M^{H}$, which we denote $\nu_{M}$, and a tubular neighborhood of $\bigcup_{H \neq\langle x\rangle \text { or }\langle y\rangle} N^{H}$, which we call $\nu_{N}$. The free $G$-manifold to which $\partial M$ is cobordant may be constructed by glueing together $\partial M-\nu\left((\partial M)^{y}\right), \partial N-\nu\left((\partial N)^{x}\right)$ and $S\left(\nu(\partial W)^{x}\right)$.

It is easy to be more explicit if we suppose now that the tubular neighborhoods $\nu_{M}$ and $\nu_{N}$ are diffeomorphic and that we can extend that diffeomorphism to include a neighborhood of $(\partial M)^{y}$ in $M$ and $(\partial N)^{x}$ in $N$. Then one may plumb $M$ and $N$ together by identifying these neghborhoods (and "rounding the corners" in a canonical way) to define a manifold with boundary whose boundary has a free $G$ action. This boundary represents the coset in $M U_{*}(B G)$ associated to $[M]$ and $[N]$ in this filtration as above.

For example, let $G=\mathbb{Z} / 2^{2}$. Given a complex representation $W$ let $\mathbb{P}(W)$ denote the space of complex one-dimensional subspaces of $W$ with inherited $G$-action. We consider $\mathbb{P}\left(\mathbb{C} \oplus V_{x}\right)$, where by abuse $\mathbb{C}$ denotes the one-dimensional trivial representation, which is just the Riemann sphere where $x$ acts trivially and $y$ acts by multiplication by -1 . Then $\mathbb{P}\left(\mathbb{C} \oplus V_{x}\right) \times D\left(V_{y}\right)$ represents a class in $\Omega_{4}^{U, G}[\mathcal{A},\langle y\rangle]$ and, 
similarly, $\mathbb{P}\left(\mathbb{C} \oplus V_{y}\right) \times D\left(V_{x}\right)$ represents a class in $\Omega_{4}^{U, G}[\mathcal{A},\langle x\rangle]$. These classes map to the same class in $\Omega_{4}^{U, G}[\mathcal{A},\langle x\rangle \cup\langle y\rangle]$ since neighborhoods of their $(x+y)$-fixed sets are both diffeomorphic to two copies of $D\left(V_{x} \oplus V_{y}\right)$. These diffeomorphisms which do extend to the boundaries as needed above. If we plumb $\mathbb{P}\left(\mathbb{C} \oplus V_{x}\right) \times D\left(V_{y}\right)$ and $\mathbb{P}\left(\mathbb{C} \oplus V_{y}\right) \times D\left(V_{x}\right)$ along these neighborhoods we get a four-manifold $P$ whose boundary is free. It is useful to picture the real analog in which two bands - $S^{1} \times D^{1}$ - are glued along $S^{0} \times I^{2}$ to get a surface diffeomorphic to $S^{2}$ with four open disks removed.

We will show in the next section the the class $[\partial P] \in M U_{3}\left(B(\mathbb{Z} / 2)^{2}\right)$ is non-zero. In fact, it must then repsent a "Tor class", by which we mean a class which is not in the image of the tensor product of $M U_{*}(B \mathbb{Z} / 2)$ with itself under the Künneth map. As far as we know, ours is the first construction of a representative for a class in $M U_{*}(B A)$ for any abelian group $A$ which is not a union of products of spheres. Our plumbing constructions greatly increase the tools with which one can create free $A$-actions.

Our techniques clearly generalize beyond $\mathbb{Z} / p^{2}$. One may chase through the ana$\log$ of Diagram 4 to define classes generated by processes composed the basic ones we have used in this section: taking boundaries, taking tubular neighborhoods of fixed sets, and using cobordisms between equivalent classes.

\section{Atiyah-Segal-Wilson Invariants}

In this section we describe some powerful invariants which may be used to study free $G$-manifolds which are boundaries of arbitrary $G$-manifolds, as are most manifolds constructed from the geometric local cohomology filtration. These invariants are essentially characteristic numbers in localized equivariant $K$-theory. We will take a geometric approach, though it will be clear how to stabilize as suggested to us by May.

Recall that in ordinary non-equivariant homology, characteristic numbers encode the homomorphism

$$
\chi: M U_{*}(X) \rightarrow H_{*}(B U \times X),
$$

which sends the fundamental class in homology of $M$ to its image under $(\tau \times f)_{*}$ in $H_{*}(B U \times X)$. When $X$ is an $H$-space (in particular, when $X$ is a point), this homomorphism is a ring homomorphism. There is a corresponding construction at the level of spectra (see for example $[\mathbf{2 7}]$ ).

Proposition 5.1. The homomorphism $\chi$ corresponds with the Boardman homomorphism

$$
h: \pi_{*}\left(M U \wedge X_{+}\right) \rightarrow \pi_{*}\left(M U \wedge H \wedge X_{+}\right) .
$$

Proof. Let $\nu$ be the normal bundle of $M$ embedded in some sphere $S^{k+N}$, and 
consider the following commutative diagram

$$
\begin{aligned}
& H_{k+N}\left(S^{k+N}\right) \stackrel{c_{*}}{\longrightarrow} H_{k+N} T(\nu) \stackrel{T(\tau \times f)_{*}}{\longrightarrow} H_{k+N}\left(T\left(\xi_{N}\right) \wedge X_{+}\right) \\
& \text {Thom } \cong \quad \text { Thom } \cong \uparrow \\
& H_{k}(M) \stackrel{(\tau \times f)_{*}}{\longrightarrow} H_{k}(B U(N) \times X),
\end{aligned}
$$

where $c$ is the collapse map onto $\nu$ as in the Pontrijagin-Thom construction. Then the top composite is the homology Boardman homomorphism, and the bottom map is $\chi$. Commutativity of the right square and the fact that $c_{*}$ is a homology isomorphism in dimension $k+N$ gives the desired correspondence between homomorphisms.

Remark. When using the natural basis for $\pi_{*}\left(M U \wedge H \wedge X_{+}\right)$these numbers correspond to the characteristic numbers of the stable normal bundle, not tangent bundle, of $M$.

A more homotopy theoretic point of view of characteristic numbers facilitates their definition for generalized, possibly equivariant, cohomology theories which have a Thom isomorphism for complex vector bundles. Given $M$ a stably complex manifold, $E$ a cohomology theory for which the Thom isomorphism theorem holds, and $x \in E^{*}(M)$ we can define "evaluation of $x$ on the fundamental class of $M$ ", as motivated by Equation 5 .

Definition 5.2. Let $x \in E^{*}(M)$. With notation as in Proposition 5.1, define the number $x[M]$ to be the image of $x$ under the composite

$$
\widetilde{E}^{*}\left(M_{+}\right) \stackrel{\cong}{\rightrightarrows} \widetilde{E}^{*+|\nu|}(T(\nu)) \stackrel{c^{*}}{\rightarrow} \widetilde{E}^{*+|\nu|}\left(S^{V}\right) \stackrel{\cong}{\rightrightarrows} E_{*-n}(p t .),
$$

where $n$ is the dimension of $M$.

This composite map goes by many names in the literature, including "Gysin map" and "wrong-way map". The class $x[M]$ is a bordism invariant of $M$ when $x$ is a characteristic class of the tangent bundle of $M$. As in the case of ordinary cohomology, these numbers encode a ring homomorphism. To avoid discussion of the fundamental class and facilitate computation, we may define this ring homomorphism by giving explicit formulae for the characteristic classes dual to multiplicative generators of $E_{*}(B U)$. We define this homomorphism now in the case of equivariant $K$-theory, which for finite complexes may be defined in an entirely analogous way to ordinary $K$-theory by using $G$-vector bundles (see [24] for a full discussion).

Remark. Equivariant $K$-theory characteristic numbers play an important role in geometry, by work of Atiyah, Segal and Singer [2]. For example if $\gamma$ is a holomorphic vector bundle over a complex $G$-manifold $M$, let $H^{i}(M, \mathcal{O}(\gamma))$ denote the complex $G$-vector space which is the cohomology of the sheaf of holomorphic sections of $\gamma$. Then the equivariant Riemann-Roch theorem of [2] states that

$$
\sum_{i}(-1)^{i} H^{i}(M, \mathcal{O}(\gamma))=\gamma[M]
$$


This sheaf cohomology will often vanish for $i>0$ so we will have that $\gamma[M]$ is isomorphic to the $G$-vector space of holomorphic sections of $\gamma$.

Definition 5.3. Let $\gamma$ be an $n$-dimensional complex $G$-vector bundle over $X$, and let $\gamma$ also denote the corresponding class in $K_{G}^{0}(X)$. The characteristic class $\beta_{i}(\gamma)$ is the coefficient of $t^{i}$ in the power series

$$
\log \left(\sum_{i \geqslant 0} \lambda^{i}(\gamma-n) \cdot t^{i}\right) \in \widetilde{K}_{G}^{0}(X)[[t]] .
$$

Recall the coefficient ring for equivariant $K$-theory, which satisfies a strong form of Bott periodicity. $K_{G}^{0}$ is isomorphic to the the representation ring $R(G)$, while $K_{G}^{1}$ is zero.

Definition 5.4. Let $M$ represent a class in $\Omega_{n}^{U, G}$. Define the ring homomorphism $\kappa: \Omega_{*}^{U, G} \rightarrow R(G)\left[\left[b_{i}\right]\right]$ by setting

$$
\kappa([M])=\sum_{I=\left(n_{1}, n_{2}, \cdots n_{k}\right)} \beta_{I}(T M)[M] \cdot b_{I}
$$

where $\beta_{I}(E)=\prod_{1 \leqslant i \leqslant k}\left(\beta_{i}(E)\right)^{n_{i}}$ and $b_{I}=b_{1}^{n_{1}} b_{2}^{n_{2}} \cdots b_{k}^{n_{k}}$.

The homomorphism $\kappa$ extends to the Boardman homomorphism $\pi_{*}\left(M U^{G}\right) \rightarrow$ $\pi_{*}\left(M U^{G} \wedge K^{G}\right)$.

We think of the coefficients of $\kappa$ as invariants of $\Omega_{*}^{U, G}$ with values in the representation ring. A standard approach to the representation ring is through character theory. We can ask what parts of the structure of a $G$-manifold $M$ we need to retain in order to understand the value of $E[M]$ at a single conjugacy class of $G$. For example, the value of $E[M]$ at the identity element, namely its dimension, will only depend on the underlying manifold $M$ and not the $G$-action. Atiyah and Segal show in [2] that the characters associated to these invariants at a single conjugacy class depend only on the fixed-set structure of $M$ at that conjugacy class, which is given by the image of $[M]$ in $\Omega_{*}^{U, H}[\mathcal{A}, \mathcal{P}]$, where $H$ is the cyclic subgroup of $G$ generated by a representative of the conjugacy class and $\mathcal{P}$ is the family of proper subgroups of $H$.

Atiyah and Segal noticed that the geometry of reduction to fixed sets coincided in algebra to localization, which we have already seen in Lemma 2.9.

Definition 5.5. Given a conjugacy class $\mathcal{C}$ of the representation $\operatorname{ring} R(G)$ let $\mathfrak{p}(\mathcal{C})$ be the prime ideal of elements of $R(G)$ whose characters do not vanish at $\mathcal{C}$. Let $R(G)_{\mathfrak{p}(\mathcal{C})}$ denote the localization of $R(G)$ at $\mathfrak{p}(\mathcal{C})$, and let $\lambda: R(G) \rightarrow R(G)_{\mathfrak{p}(\mathcal{C})}$ denote the canonical map of $R(G)$ into this localization.

Definition 5.6. Let $e v_{\mathcal{C}}: R(G)_{\mathfrak{p}(\mathcal{C})} \rightarrow \mathbb{C}$ denote the map which gives the value of a localized character at $\mathcal{C}$. This map is well-defined because characters of denominators in $R(G)_{\mathfrak{p}(\mathcal{C})}$ do not vanish on $\mathcal{C}$. 
Theorem 5.7 (Atiyah-Segal). Let $\mathcal{C}$ be a conjugacy class of $G$ and let $h \in \mathcal{C}$. Then the composite

$$
\Omega_{*}^{U, G} \stackrel{\kappa}{\rightarrow} R(G)\left[\left[b_{i}\right]\right] \stackrel{\lambda}{\rightarrow} R(G)_{\mathfrak{p}(\mathcal{C})}\left[\left[b_{i}\right]\right] \stackrel{e v_{\mathcal{C}}}{\longrightarrow} \mathbb{C}\left[\left[b_{i}\right]\right]
$$

factors though the homomorphism $\Omega_{*}^{U, G} \rightarrow \Omega_{*}^{U, H}[\mathcal{A}, \mathcal{P}]$. In other words, there is a homomorphism

$$
\kappa_{\mathcal{C}}: \Omega_{*}^{U, H}[\mathcal{A}, \mathcal{P}] \rightarrow \mathbb{C}\left[\left[b_{i}\right]\right]
$$

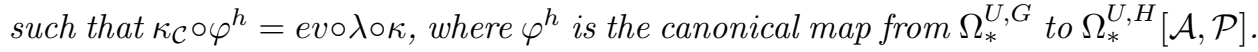

We will give a formula for $\kappa_{\mathcal{C}}$ below.

Now note that the maps from $\Omega_{*}^{U, G}$ to $\Omega_{*}^{U, H}[\mathcal{A}, \mathcal{P}]$ all factor through $\Omega_{*}^{U, G}[\mathcal{A}, e]$. This observation in conjunction with Theorem 5.7 leads to powerful invariants for $M U_{*}(B G)$. Consider the following diagram

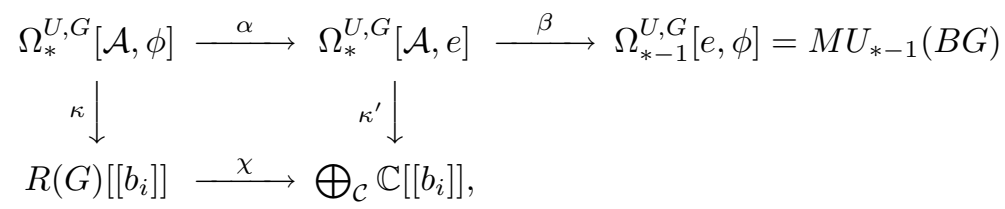

where by abuse we are now using $\chi$ to denote the character map in representation theory. By exactness of the first row and commutativity of the square we may deduce the following.

Theorem 5.8. If class $[M, \partial M] \in \Omega_{*}^{U, G}[\mathcal{A}, e]$ has image under $\kappa^{\prime}$ which is not in the image of $\chi$ then $[\partial M]$ is non-zero $M U_{*-1}(B G)$.

In $[\mathbf{2 9}]$, G. Wilson shows that the converse of this theorem is true for groups with periodic cohomology.

We now present the cohomological definition of $\kappa_{\mathcal{C}}$, as given in [2]. First note that by Proposition 2.8 an element $[M]$ of $\Omega_{*}^{U, H}[\mathcal{A}, \mathcal{P}]$ is represented by a tubular neighborhood of $M^{H}$, which by the equivariant tubular neighborhood theorem is diffeomorphic to the total space of an $H$ vector bundle over $M^{H}$. We will be viewing classes as represented by such bundles for the purposes of defining our fomulae. We first define an equivariant version of the Chern character for $G$-bundles over trivial $G$-spaces.

Definition 5.9. Let $E$ be a complex $G$-vector bundle over a trivial $G$-space $X$, which decomposes as $\oplus_{\rho_{i} \in \operatorname{Irr}(G)} E_{\rho_{i}}$ (all such $G$-bundles do so - see [24]). For each $g \in G$ define

$$
\operatorname{ch}(E)(g)=\sum_{\rho_{i} \in \operatorname{Irr}(G)} \chi_{\rho_{i}}(g) \cdot \operatorname{ch}\left(E_{\rho_{i}}\right),
$$

where $\chi_{\rho_{i}}(g)$ is the trace of $g$ acting on $\rho_{i}$.

We next introduce the Todd class which is needed to translate from $K$-theory to cohomology. Let $c_{i}$ denote the $i$ th Chern class of a complex vector bundle $\gamma$ in $H^{2 i}(X)$. Form the ring

$$
H^{*}(X)\left[a_{1}, \cdots, a_{n}\right] /\left(s_{i}\left(a_{1}, \cdots, a_{n}\right)=c_{i}\right),
$$


where $s_{i}$ denotes the $i$ th elementary symmetric polynomial in the variables indicated. Thus, any symmetric polynomial in the $a_{i}$ will be a polynomial in the $c_{i}$.

Definition 5.10. Let $M$ be a unitary manifold. The Todd class of $M$, denoted $\operatorname{td}(M)$, is defined by taking $\gamma$ to be a complex vector bundle which is a lift of the stable tangent bundle of $M$. Then set

$$
t d(M)=\prod_{i=1}^{n} \frac{a_{i}}{1-e^{-a_{i}}} .
$$

Now let $H$ be a cyclic group of order $n$, and fix a generator $h$ of $H$. Let $\zeta=e^{\left(\frac{2 \pi i}{n}\right)}$. And let $\rho_{i}$ be the representation of $H$ in which the generator $h$ acts on $\mathbb{C}$ by multiplication by $\zeta^{i}$.

Definition 5.11. Let $E_{\rho_{i}}$ be a complex $H$-vector bundle over a base with trivial $H$-action and fiber isomorphic to $\oplus_{k} \rho_{i}$ for some $k$. Define

$$
\mathcal{U}_{i}\left(E_{\rho_{i}}\right)=\prod_{1 \leqslant j \leqslant k}\left(\frac{\zeta^{i}-1}{\zeta^{i}-e^{-a_{j}}}\right) .
$$

If $E=\oplus_{1 \leqslant j<n} E_{\rho_{i}}$. Define

$$
\mathcal{U}(E)=\prod_{i} \mathcal{U}_{i}\left(E_{\rho_{i}}\right)
$$

We are now ready to present the cohomological formula we will use to define the $\operatorname{maps} \kappa_{\mathcal{C}}$.

Definition 5.12. Let $H$ be a cyclic group, generated by $h$. Let $X \in \Omega_{*}^{U, H}[\mathcal{A}, \mathcal{P}]$ be represented by a $G$-bundle whose base is $M$, a stably complex manifold, whose total space we denote $\nu$ and whose fiber is isomorphic to a representation $V$ such that $V^{H}=0$. Let $E$ be an element of $K_{H}^{0}(M)$. Define

$$
E[X]=\left\{\frac{\operatorname{ch}(E)(h) \cdot \mathcal{U}(\nu) \cdot t d(M)}{\operatorname{det}\left(1-h \mid V^{*}\right)}\right\}[M]
$$

Definition 5.13. Let $\mathcal{C}$ be a conjugacy class of $G$. Let $h \in \mathcal{C}$, and let $H$ be the cyclic subgroup generated by $h$. Let $X$ represent a class in $\Omega_{n}^{U, H}[\mathcal{A}, \mathcal{P}]$. Define the ring homomorphism $\kappa_{\mathcal{C}}: \Omega_{*}^{U, H}[\mathcal{A}, \mathcal{P}] \rightarrow R(G)_{\mathfrak{p}(\mathcal{C})}\left[\left[b_{i}\right]\right]$ by

$$
\kappa_{\mathcal{C}}([X])=\sum_{I=\left(n_{1}, n_{2}, \cdots n_{k}\right)} \beta_{I}(T X-n)[X] \cdot b_{I}
$$

where $\beta_{I}(E)=\prod_{1 \leqslant i \leqslant k}\left(\beta_{i}(E)\right)^{n_{i}}$ and $b_{I}=b_{1}^{n_{1}} b_{2}^{n_{2}} \cdots b_{k}^{n_{k}}$.

Though the formula in Definition 5.12 looks complicated, it is manageable in some cases, especially when the fixed sets are isolated points.

Example 1. Let $G=\mathbb{Z} / p$ and fix a generator $g$. Consider the class $X_{1, \rho_{1}} \in$ $\Omega_{2}^{U, G}[\mathcal{A}, e]$ represented by $D\left(\rho_{1}\right)$. In the notation of Definition 5.12 , for $X_{1, \rho_{1}}$ we have 
$V=\rho_{1}, \nu$ is a trivial bundle, and $M$ is a point. Substituting, $\mathcal{U}(\nu)=t d(M)=1$, so that

$$
\kappa_{g}\left(X_{1, \rho_{1}}\right)=\frac{1}{1-\zeta^{-1}} \cdot f\left(b_{i}\right),
$$

where $f$ is a power series in $b_{i}$ whose coefficients lie in $\mathbb{Z}[\zeta]$ and whose constant term is one.

Example 2. Let $G=\mathbb{Z} / 4$ and fix a generator $g$. Consider the complex $G$-manifold with free boundary $X$ whose underlying topology is that of two complex disks, where the generator $g$ acts by mapping the disks to one another so that $g^{2}$ acts by multiplying by -1 on each disk. Then since $g$ and $g^{3}$ do not fix any points of $X$, we have $\kappa_{g}(X)=\kappa_{g^{3}}(X)=0$. Restricted to $\left\langle g^{2}\right\rangle, X$ is two copies of $X_{1, \rho_{1}}$, where $\rho_{1}$ is the sign representation of $\mathbb{Z} / 2$. By the above example $\kappa_{g^{2}}(X)$ is integral with constant term one. Thus, from the constant terms of the $\kappa_{\mathcal{C}}(X)$ we construct a localized character whose values are $(0,1,0)$ at $g, g^{2}$ and $g^{3}$, respectively. Even though each entry of this localized character is an integer, the character itself is not integral in that it is not in the span of $(1,1,1),(i,-1,-i),(-1,1,-1)$ and $(-i,-1, i)$, the values of characters of irreducible representations of $G$ at $g, g^{2}$ and $g^{3}$. Thus $X$ is not the fixed set of any complex $\mathbb{Z} / 4$ surface.

Finally, we apply these invariants to the $\mathbb{Z} / 2^{2}$ manifold $P$ defined at the end of the previous section. $P$ has two fixed points, whose normal bundles are $V_{x} \oplus V_{y}$ in the notation of the previous section. If we look at the conjugacy class of $x+y$, there are only these two fixed points and the computations proceed as in the first example above to give a value of $1 / 4+1 / 4=1 / 2$ multiplied by some integral power series in $b_{i}$, which shows that the boundary of $P$ represents a non-trivial class in $M U_{3}\left(B \mathbb{Z} / 2^{2}\right)$. At the conjugacy class $x$ (respectively $y$ ) the fixed set is a $\mathbb{P}^{1}$, whose Todd genus is $1+c_{1}\left(\mathbb{P}^{1}\right) / 2=1+a$, where $a$ is the generator of $H^{2}$. As in the first example, there is a two in the denominator coming from the action of $x$ on the normal bundle, and so we once again get that the value of the localized character is $1 / 2$ multiplied by an integral series.

\section{Directions for Further Work}

We leave with a couple suggestions for projects which may use these techniques.

- Do computations in the local cohomology spectral sequence for $M U_{*}(B A)$. Compare this filtration to the Künneth filtration (they seem to be very closely related), which is one of the main tools for computations so far [17]. AtiyahSegal-Wilson invariants may be useful in solving extension problems.

- Translate these constructions to the Spin setting in order to approach the Gromov-Lawson-Rosenberg conjecture for finite groups $[\mathbf{3}, \mathbf{2 6}]$. Part of the approach of $[\mathbf{3}]$ is to find arbitrary $G$-manifolds whose boundaries generate free $G$-bordism. 


\section{References}

[1] J.F. Adams. Prerequisites (in Equivariant Stable Homotopy Theory) for Carlsson's Lecture. In Algebraic Topology, Aarhus 1982, Volume 1051 of Lecture Notes in Mathematics, Springer, Berlin, New-York, 1984.

[2] M.F. Atiyah, G.B. Segal and I. Singer. The Index of Elliptic Operators II, III. Annals of Mathematics 87 (1968), 531-578

[3] B. Botvinnik, P. Gilkey, S. Stolz. The Gromov-Lawson-Rosenberg conjecture for groups with periodic cohomology. Journal of Differential Geometry 46 (1997), no. 3, 374-405.

[4] T. Brcker and E. Hook. Stable equivariant bordism. Math. Z. 129 (1972), 269-277

[5] B. Bruner and J.P.C. Greenlees. Connective $K$-theory of finite groups. Preprint, 2001.

[6] G. Carlsson. A Survey of Equivariant Stable Homotopy Theory. Topology 31 (1992), 1-27.

[7] P.E. Conner and E.E. Floyd. Differentiable Periodic Maps. Springer, BerlinHeidelberg-New York, 1964.

[8] S. Costenoble. Chapter 15 of [22].

[9] T. tom Dieck. Bordism of $G$-Manifolds and Integrality Theorems. Topology 9 (1970), 345-358.

[10] T. Goodwillie. Calculus II: Analytic Functors. K-Theory 5 (1992) 295-332.

[11] J.P.C. Greenlees. $K$-homology of universal spaces and local cohomology of the representation ring. Topology 32 (1993), 295-308.

[12] J.P.C. Greenlees. Local cohomology in equivariant topology. Preprint, 2000.

[13] J.P.C. Greenlees and H. Sadofsky. Tate cohomology of theories with onedimensional coefficient ring. Topology 37 (1998) 145-174.

[14] J.P.C. Greenlees and J.P. May. Localization and completion theorems for MU-Module Spectra. Annals of Mathematics 146 (1997) 509-544.

[15] J.P.C. Greenlees and J.P. May. Generalized Tate cohomology. Memoir of the American Mathematical Society \#543, 1995.

[16] R. Harthshorne. Local Cohomology. Lecture Notes in Mathematics, No. 41, (1967).

[17] D.C. Johnson, W.S. Wilson, and D.Y. Yan. Brown-Peterson homology of elementary p-groups, II. Topology and Its Applications, 59 (1994) 117-136.

[18] I. Kriz. On Complex Equivariant Bordism Rings for $G=\mathbb{Z} / p$. To appear in Proceedings of Conference in Honor of Michael Boardman

[19] P. Landweber. Cobordism and classifying spaces. Proc. Symp. Pure Math. 22 (1971), 125-129.

[20] P. Landweber. Unitary bordism of cyclic group actions. Proceedings of the AMS 31 (1972) 564-570. 
[21] I. Madsen and R.J. Milgram. Classifying Spaces for Surgery and Cobordism of Manifolds. Volume 92 of Annals of Mathematics Studies. Princeton University Press, Princeton, 1979.

[22] J.P. May et. al. Equivariant Homotopy and Cohomology Theory. Volume 91 of the CBMS Regional Conference Series in Mathematics. AMS Publications, Providence, 1996.

[23] D. Ravenel. Nilpotence and Periodicity in Stable Homotopy Theory. Volume 126 of Annals of Mathematicas Studies. Princeton University Press, Princeton, 1992.

[24] G. Segal. Equivariant K-Theory. Publications of the IHES 34 (1968), 129 151.

[25] D.P. Sinha. Computations of Complex Equivariant Bordism Rings. To appear in the American Journal of Mathematics, 2001.

[26] S. Stolz. Simply connected manifolds of positive scalar curvature. Annals of Mathematics (2) 136 (1992) 511-540.

[27] R.E. Stong. Notes on Cobordism Theory. Princeton University Press, Princeton, 1968.

[28] S. Waner. Equivariant $R O(G)$-graded bordism theories. Topology and its $A p$ plications 17 (1984), 1-26.

[29] G. Wilson. K-Theory Invariants for Unitary G-Bordism. Quarterly Journal of Mathematics Oxford (2) 24 (1973), 499-526

This article may be accessed via WWW at http://www.rmi.acnet.ge/hha/ or by anonymous ftp at

ftp://ftp.rmi.acnet.ge/pub/hha/volumes/2001/n2a6/v3n2a6.(dvi,ps,pdf)

Dev P. Sinha dps@math.brown.edu

Department of Mathematics

Brown University

Providence, RI 02906 